\title{
Junctional Epidermolysis Bullosa
}

National Cancer Institute

\section{Source}

National Cancer Institute. Junctional Epidermolysis Bullosa. NCI Thesaurus. Code C90598.

Epidermolysis bullosa characterized by separation of the skin through the lamina lucida resulting in blister formation. It includes lethal and non-lethal variants. 